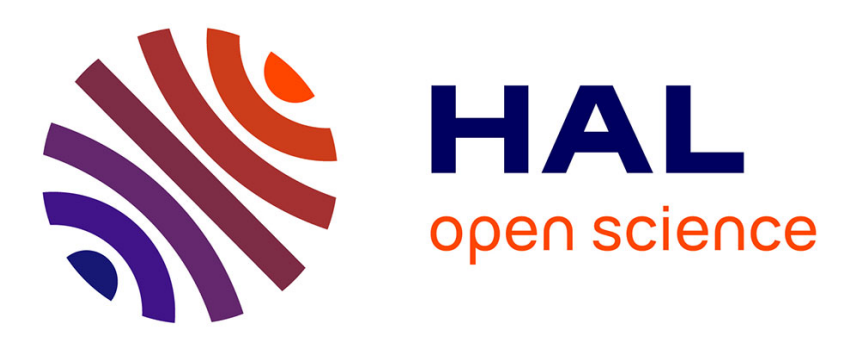

\title{
Learning Prime Implicant Conditions From Interpretation Transition
}

\author{
Tony Ribeiro, Katsumi Inoue
}

\section{To cite this version:}

Tony Ribeiro, Katsumi Inoue. Learning Prime Implicant Conditions From Interpretation Transition. The 24th International Conference on Inductive Logic Programming (ILP 2014), Sep 2015, Nancy, France. hal-01710486

\author{
HAL Id: hal-01710486 \\ https://hal.science/hal-01710486
}

Submitted on 16 Feb 2018

HAL is a multi-disciplinary open access archive for the deposit and dissemination of scientific research documents, whether they are published or not. The documents may come from teaching and research institutions in France or abroad, or from public or private research centers.
L'archive ouverte pluridisciplinaire HAL, est destinée au dépôt et à la diffusion de documents scientifiques de niveau recherche, publiés ou non, émanant des établissements d'enseignement et de recherche français ou étrangers, des laboratoires publics ou privés. 


\title{
Learning Prime Implicant Conditions From Interpretation Transition
}

\author{
Tony Ribeiro ${ }^{1}$ and Katsumi Inoue ${ }^{1,2}$ \\ 1 The Graduate University for Advanced Studies (Sokendai), \\ 2-1-2 Hitotsubashi, Chiyoda-ku, Tokyo 101-8430, Japan \\ tony_ribeiro@nii.ac.jp, \\ 2 National Institute of Informatics, \\ 2-1-2 Hitotsubashi, Chiyoda-ku, Tokyo 101-8430, Japan, \\ inoue@nii.ac.jp
}

\begin{abstract}
In a previous work we proposed a framework for learning normal logic programs from transitions of interpretations. Given a set of pairs of interpretations $(I, J)$ such that $J=T_{P}(I)$, where $T_{P}$ is the immediate consequence operator, we infer the program $P$. Here we propose a new learning approach that is more efficient in terms of output quality. This new approach relies on specialization in place of generalization. It generates hypotheses by specialization from the most general clauses until no negative transition is covered. Contrary to previous approaches, the output of this method does not depend on variables/transitions ordering. The new method guarantees that the learned rules are minimal, that is, the body of each rule constitutes a prime implicant to infer the head.
\end{abstract}

Keywords: dynamical systems, Boolean networks, attractors, supported models, learning from interpretation, Inductive Logic Programming

\section{Introduction}

In recent years, there has been a notable interest in the field of Inductive Logic Programming (ILP) to learn from system state transitions as part of a wider interest in learning the dynamics of systems $[14,2,4,8]$. Learning system dynamics has many applications in multi-agent systems, robotics and bioinformatics alike. Knowledge of system dynamics can be used by agents and robots for planning and scheduling. In bioinformatics, learning the dynamics of biological systems can correspond to the identification of the influence of genes and can help to design more efficient drugs. In some previous works, state transition systems are represented with logic programs [6,9], in which the state of the world is represented by an Herbrand interpretation and the dynamics that rule the environment changes are represented by a logic program $P$. The rules in $P$ specify the next state of the world as an Herbrand interpretation through the immediate consequence operator (also called the $T_{P}$ operator) $[18,1]$. With such a background, Inoue et al. [8] have recently proposed a framework to learn logic 
programs from traces of interpretation transitions (LFIT). The learning setting of this framework is as follows. We are given a set of pairs of Herbrand interpretations $(I, J)$ as positive examples such that $J=T_{P}(I)$, and the goal is to induce a normal logic program (NLP) $P$ that realizes the given transition relations. In [8], the authors showed one of the possible usages of LFIT: LF1T, learning from 1-step transitions. In that paper, an algorithm is proposed to iteratively learn an NLP that realizes the dynamics of the system by considering step transitions one by one. The iterative character of LF1T has applications in bioinformatics, cellular automata, multi-agent systems and robotics.

In this paper, our main concern is the minimality of the rules and the NLPs learned by LF1T. Our goal is to learn all minimal conditions that imply a variable to be true in the next state, e.g. all prime implicant conditions. In bioinformatics, for a gene regulatory network, it corresponds to all minimal conditions for a gene to be activated/inhibited. It can be easier and faster to perform model checking on Boolean networks represented by a compact NLP than the set of all state transitions. Knowing the minimal conditions required to perform the desired state transitions, a robot can optimize its actions to achieve its goals with less energy consumption. From a technical point of view, for the sake of memory usage and reasoning time, a small NLP could also be preferred in multi-agent and robotics applications. For this purpose, we propose a new version of the LF1T algorithm based on specialization. Specialization is usually considered the dual of generalization in ILP $[13,11,12]$. Where generalization occurs when a hypothesis does not explain a positive example, specialization is used to refine a hypothesis that implies a negative example.

In [7], prime implicants are defined for DNF formula as follows: a clause $C$, implicant of a formula $\phi$, is prime if and only if none of its proper subset $S \subset C$ is an implicant of $\phi$. In this work, explanatory induction is considered, while in our approach prime implicants are defined in the LFIT framework. Knowing the Boolean functions, prime implicants could be computed by Tisons consensus method [17] and its variants [10]. The novelty of our approach, is that we compute prime implicants incrementally during the learning of the Boolean function. In [8, 16], LF1T uses resolution techniques to generalize rules and reduces the size of the output NLP. This technique generates hypotheses by generalization from the most specific clauses until every positive transitions are covered. Compared to previous LF1T algorithms, the novelty of our new approach is that it generates hypotheses by specialization from the most general clauses until no negative transition is covered. The main weak point of the previous LF1T algorithms is that the output NLPs depends on variable/transition ordering. Our new method guarantees that the NLPs learned contain only minimal conditions for a variable to be true in the next state. Study of the computational complexity of our new method shows that it remains equivalent to the previous version of LF1T. Using examples from the biological literature, we show through experimental results that our specialization method can compete with the previous versions of LF1T in practice. We provide all proofs of theorems in the appendix. 


\section{Background}

In this section we recall some preliminaries of logic programming. We consider a first-order language and denote the Herbrand base (the set of all ground atoms) as $\mathcal{B}$. A (normal) logic program (NLP) is a set of rules of the form

$$
A \leftarrow A_{1} \wedge \cdots \wedge A_{m} \wedge \neg A_{m+1} \wedge \cdots \wedge \neg A_{n}
$$

where $A$ and $A_{i}$ 's are atoms $(n \geq m \geq 0)$. For any rule $R$ of the form (1), the atom $A$ is called the head of $R$ and is denoted as $h(R)$, and the conjunction to the right of $\leftarrow$ is called the body of $R$. We represent the set of literals in the body of $R$ of the form (1) as $b(R)=\left\{A_{1}, \ldots, A_{m}, \neg A_{m+1}, \ldots, \neg A_{n}\right\}$, and the atoms appearing in the body of $R$ positively and negatively as $b^{+}(R)=\left\{A_{1}, \ldots, A_{m}\right\}$ and $b^{-}(R)=\left\{A_{m+1}, \ldots, A_{n}\right\}$, respectively. The set of ground instances of all rules in a logic program $P$ is denoted as $\operatorname{ground}(P)$.

The Herbrand Base of a program $P$, denoted by $\mathcal{B}$, is the set of all atoms in the language of $P$. An interpretation is a subset of $\mathcal{B}$. If an interpretation is the empty set, it is denoted by $\epsilon$. An interpretation $I$ is a model of a program $P$ if $b^{+}(R) \subseteq I$ and $b^{-}(R) \cap I=\emptyset$ imply $h(R) \in I$ for every rule $R$ in $P$. For a logic program $P$ and an Herbrand interpretation $I$, the immediate consequence operator (or $T_{P}$ operator) [1] is the mapping $T_{P}: 2^{\mathcal{B}} \rightarrow 2^{\mathcal{B}}$ :

$$
T_{P}(I)=\left\{h(R) \mid R \in \operatorname{ground}(P), b^{+}(R) \subseteq I, b^{-}(R) \cap I=\emptyset\right\} .
$$

In the rest of this paper, we only consider rules of the form (1). To simplify the discussion we will just use the term rule.

Definition 1 (Subsumption). Let $R_{1}$ and $R_{2}$ be two rules. If $h\left(R_{1}\right)=h\left(R_{2}\right)$ and $b\left(R_{1}\right) \subseteq b\left(R_{2}\right)$ then $R_{1}$ subsumes $R_{2}$. If $b\left(R_{1}\right) \subset b\left(R_{2}\right)$ then $R_{1}$ is more general than $R_{2}$ and $R_{2}$ is more specific than $R_{1}$. Let $S$ be a set of rules and $R$ be a rule. If there exists a rule $R^{\prime} \in S$ that subsumes $R$ then $S$ subsumes $R$. This also holds for normal logic program since a NLP is a set of rules.

In ILP, search mainly relies on generalization and specialization that are dual notions. Generalization is usually considered an induction operation, and specialization a deduction operation. In [13], the author define the minimality and maximality of the generalization and specialization operations as follows.

Definition 2 (Generalization operator [13]). A generalization operator maps a conjunction of clauses $S$ onto a set of minimal generalizations of $S$. A minimal generalization $G$ of $S$ is a generalization of $S$ such that $S$ is not a generalization of $G$, and there is no generalization $G^{\prime}$ of $S$ such that $G$ is a generalization of $G^{\prime}$.

Definition 3 (Specialization operator [13]). A specialization operator maps a conjunction of clauses $G$ onto a set of maximal specializations of $G$. A maximal specialization $S$ of $G$ is a specialization of $G$ such that $G$ is not a specialization of $S$, and there is no specialization $S^{\prime}$ of $G$ such that $S$ is a specialization of $S^{\prime}$. 
The body of a rule of the form (1) can be considered as a clause, so that, the Definition 2 and 3 can also be used to compare the body of two rules.

\section{Learning from 1-Step Transitions}

LF1T is an any time algorithm that takes a set of one-step state transitions $E$ as input. These one-step state transitions can be regarded as positive examples. From these transitions the algorithm learns a logic program $P$ that represents the dynamics of $E$. To perform this learning process we can iteratively consider onestep transitions. Figure 1 represents a Boolean nework with its corresponding state transition diagram. Given this state transition diagram as input, LF1T can learn the Boolean network $N_{1}$.

In $L F 1 T$, the Herbrand base $\mathcal{B}$ is assumed to be finite. In the input $E$, a state transition is represented by a pair of Herbrand interpretations. The output of $L F 1 T$ is an NLP that realizes all state transitions of $E$.

\section{Learning from 1-Step Transitions (LF1T)}

Input: $\quad E \subseteq 2^{\mathcal{B}} \times 2^{\mathcal{B}}$ : (positive) examples/observations

Output: An NLP $P$ such that $J=T_{P}(I)$ holds for any $(I, J) \in E$.

To construct an NLP with LF1T we use a bottom-up method that generates hypotheses by generalization from the most specific clauses or examples until every positive example is covered. LF1T first constructs the most specific rule $R_{A}^{I}$ for each positive literal $A$ appearing in $J=T_{P}(I)$ for each $(I, J) \in E$ :

$$
R_{A}^{I}:=\left(A \leftarrow \bigwedge_{B_{i} \in I} B_{i} \wedge \bigwedge_{C_{j} \in \mathcal{B} \backslash I} \neg C_{j}\right)
$$

It is important here that we do not construct any rule to make a literal false. For instance, from the state transition $(q r, p r)$ LF1T will learn only two rules: $p \leftarrow \neg p \wedge q \wedge r$ and $r \leftarrow \neg p \wedge q \wedge r$. The rule $R_{A}^{I}$ is then possibly generalized when another transition from $E$ makes $A$ true, which is computed by several generalization methods.

The two generalization methods considered in [8] are based on resolution. In [8], naïve and ground resolutions are defined between two ground rules as follows. Let $R_{1}, R_{2}$ be two ground rules and $l$ be a literal such that $h\left(R_{1}\right)=h\left(R_{2}\right)$,

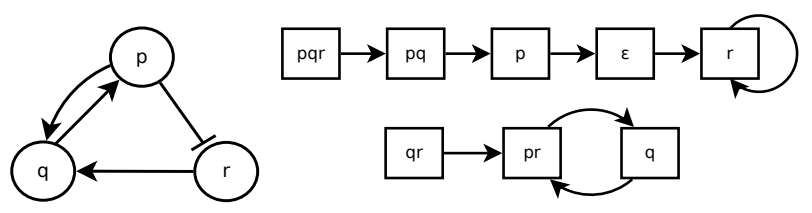

Fig. 1. A Boolean Network $N_{1}$ (left) and its state transition diagram (right) 
$l \in b\left(R_{1}\right)$ and $\bar{l} \in b\left(R_{2}\right)$. If $\left(b\left(R_{2}\right) \backslash\{\bar{l}\}\right) \subseteq\left(b\left(R_{1}\right) \backslash\{l\}\right)$ then the ground resolution of $R_{1}$ and $R_{2}$ (upon $l$ ) is defined as

$$
\operatorname{res}\left(R_{1}, R_{2}\right)=\left(h\left(R_{1}\right) \leftarrow \bigwedge_{L_{i} \in b\left(R_{1}\right) \backslash\{l\}} L_{i}\right) .
$$

In particular, if $\left(b\left(R_{2}\right) \backslash\{\bar{l}\}\right)=\left(b\left(R_{1}\right) \backslash\{l\}\right)$ then the ground resolution is called the naïve resolution of $R_{1}$ and $R_{2}$ (upon $l$ ). In this particular case, the rules $R_{1}$ and $R_{2}$ are said to be complementary to each other with respect to $l$.

Both naïve resolution and ground resolution can be used as generalization methods of ground rules. For two ground rules $R_{1}$ and $R_{2}$, the naïve resolution $\operatorname{res}\left(R_{1}, R_{2}\right)$ subsumes both $R_{1}$ and $R_{2}$, but the non-naïve ground resolution subsumes $R_{1}$ only.

Definition 4 (Consistency). Let $R$ be a rule and $E$ be a set of state transitions. $R$ is consistent with $E$ if $\forall(I, J) \in E$ when $b(R) \subseteq I$ then $h(R) \in J$. Let $P$ be a NLP. $P$ is consistent with $E$ if $\forall(I, J) \in E, \forall R^{\prime} \in P$, when $b\left(R^{\prime}\right) \subseteq I$ then $h\left(R^{\prime}\right) \in J$.

Ground and naïve resolutions can be used to learn a ground NLP. Both methods keep the consistency of the learned rules. For example, let us consider the three rules: $R_{1}=(p \leftarrow q \wedge r), R_{2}=(p \leftarrow \neg q \wedge r), R_{3}=(p \leftarrow \neg q)$, and their resolvent: $\operatorname{res}\left(R_{1}, R_{2}\right)=\operatorname{res}\left(R_{1}, R_{3}\right)=(p \leftarrow r)$. $R_{1}$ and $R_{2}$ are complementary with respect to $q$. Both $R_{1}$ and $R_{2}$ can be generalized by the naïve resolution of them because res $\left(R_{1}, R_{2}\right)$ subsumes both $R_{1}$ and $R_{2}$. On the other hand, the ground resolution $\operatorname{res}\left(R_{1}, R_{3}\right)$ of $R_{1}$ and $R_{3}$ is equivalent to $\operatorname{res}\left(R_{1}, R_{2}\right)$. However, $\operatorname{res}\left(R_{1}, R_{3}\right)$ subsumes $R_{1}$ but does not subsume $R_{3}$.

LF1T with Naïve Resolution: In the first implementation of LF1T of [8], naïve resolution is used as a least generalization method. This method is particularly intuitive from the ILP viewpoint, since each generalization is performed based on a least generalization operator. In [8], it is shown that for two complementary ground rules $R_{1}$ and $R_{2}$, the naïve resolution of $R_{1}$ and $R_{2}$ is the least generalization [15] of them, that is, $\lg \left(R_{1}, R_{2}\right)=\operatorname{res}\left(R_{1}, R_{2}\right)$. When naïve resolution is used, LF1T needs an auxiliary set $P_{\text {old }}$ of rules to globally store subsumed rules, which increases monotonically. $P_{\text {old }}$ is set to be $\emptyset$ at first. When a generated rule $R$ is newly added LF1T searches a rule $R^{\prime} \in P \cup P_{\text {old }}$ such that $h\left(R^{\prime}\right)=h(R)$ and $b(R)$ and $b\left(R^{\prime}\right)$ differ in the sign of only one literal $l$. If there is no such a rule $R^{\prime}$, then $R$ is just added to $P$; otherwise, $R$ and $R^{\prime}$ are added to $P_{\text {old }}$ and then $\operatorname{res}\left(R, R^{\prime}\right)$ is added to $P$.

LF1T with Ground Resolution: Using naïve resolution, $P \cup P_{\text {old }}$ possibly contains all patterns of rules constructed from the Herbrand base $\mathcal{B}$ in their bodies. In the second implementation of LF1T of [8], ground resolution is used as an alternative generalization method. This replacement of resolution leads to a lot of computational gains since the use of $P_{\text {old }}$ is not necessary any more: all generalized rules obtained from $P \cup P_{\text {old }}$ by naïve resolution can be obtained using ground resolution on $P$. By Theorem 3 of [8], using the naïve version, 
the memory use of the LF1T algorithm is bounded by $O\left(n \cdot 3^{n}\right)$, and the time complexity of learning is bounded by $O\left(n^{2} \cdot 9^{n}\right)$, where $n=|\mathcal{B}|$. On the other hand, with ground resolution, the memory use is bounded by $O\left(2^{n}\right)$, which is the maximum size of $P$, and the time complexity is bounded by $O\left(4^{n}\right)$. Given the set $E$ of complete state transitions, which has the size $O\left(2^{n}\right)$, the complexity of $\operatorname{LF1T}(E, \emptyset)$ with ground resolution is bounded by $O\left(|E|^{2}\right)$. On the other hand, the worst-case complexity of learning with naïve resolution is $O\left(n^{2} \cdot|E|^{4.5}\right)$.

\section{Learning Prime Implicant Conditions}

In this section, we use the notion of prime implicant to define minimality of NLP. We consider that the NLP learn by LF1T is minimal if the body of each rule constitutes a prime implicant to infer the head.

Definition 5 (Prime Implicant Condition). Let $R$ be a rule and $E$ a set of state transitions such that $R$ is consistent with $E . b(R)$ is a prime implicant condition of $h(R)$ for $E$ if there does not exist another rule $R^{\prime}$ consistent with $E$ such that $R^{\prime}$ subsumes $R$. Let $P$ be a NLP such that $P \cup\{R\} \equiv P$ : all models of $P \cup\{R\}$ are models of $P$ and vice versa. $b(R)$ is a prime implicant condition of $h(R)$ for $P$ if there does not exist another rule $R^{\prime}$ such that $P \cup\left\{R^{\prime}\right\} \equiv P$ and $R^{\prime}$ subsumes $R$.

Definition 6 (Prime Rule). Let $R$ be a rule and $E$ a set of state transitions such that $R$ is consistent with $E$. Let $P$ be a NLP such that $P \cup\{R\} \equiv P$. R is a prime rule of $E$ (resp. $P$ ) if $b(R)$ is a prime implicant condition of $h(R)$ for $E$ (resp. $P$ ). For any atom $p$ the most general prime rule for $p$ is the rule with an empty body $(p \leftarrow)$ that states that $p$ is always true in the next state.

Example 1. Let $R_{1}, R_{2}$ and $R_{3}$ be three rules and $E$ be the set of state transitions of Figure 1 as follows: $R_{1}=p \leftarrow p \wedge q \wedge r, R_{2}=p \leftarrow p \wedge q, R_{3}=p \leftarrow q$ The only rule more general than $R_{3}$ is $R^{\prime}=p$., but $R^{\prime}$ is not consistent with $(p, \epsilon) \in E$ so that $R_{3}$ is a prime rule for $E$. Since $R_{3}$ subsumes both $R_{1}$ and $R_{2}$, they are not prime rules of $E$. Let $P$ be the NLP $\{p \leftarrow p, q \leftarrow p \wedge r, r \leftarrow \neg p\}, R_{3}$ is a prime rule of $P$ because $P$ realizes $E$ and $R_{3}$ is minimal for $E$.

Definition 7 (Prime NLP). Let $P$ be an NLP and $E$ be the state transitions of $P, P$ is a prime NLP for $E$ if $P$ realizes $E$ and all rules of $P$ are prime rule for $E$. We call the set of all prime rules of $E$ the complete prime NLP of $E$.

Example 2. Let $R_{1}, R_{2}$ and $R_{3}$ be three rules, $E$ be the set of state transitions of Figure 1 and $P$ an NLP as follows: $R_{1}=p \leftarrow p \wedge q, R_{2}=q \leftarrow p \wedge r, R_{3}=r \leftarrow \neg p$ and $P=\left\{R_{1}\right\} \cup\left\{R_{2}\right\} \cup\left\{R_{3}\right\}$. Since $R_{1}, R_{2}$ and $R_{3}$ are prime rule for $E, P$ the NLP formed of these three rules is a prime NLP of $E$. There does not exist any other prime rules for $E$, therefore $P$ is also the complete prime NLP of $E$.

The complete prime NLP of a given set of state transitions $E$ can naïvely be obtained by brute force search. Starting from the most general rules, that is, 
fact rules, it suffices to generate all maximal specific specialization step by step and keep the first ones that are consistent with $E$. This method implies to check all state transitions for all possible rules that correspond to $O\left(n \times 3^{n} \times 2^{n}\right)=$ $O\left(6^{n}\right)$ checking operations in the worst case for a Herbrand base of $n$ variables. But it is also possible to do it by extending previous LF1T algorithm for the sake of complexity. Here we propose a simple extension of naïve (resp. ground) resolution. In previous algorithms, for each rule learned, only the first least generalization found is kept. Now we consider all possible least generalizations and define full naïve (resp. ground) resolution. LF1T with full naïve (resp. ground) resolution learn the complete prime NLP that realize the input state transitions.

Definition 8 (full naive resolution and full ground resolution). Let $R$ be a rule and $P$ be a NLP. Let $P_{R}$ be a set of rule of $P$ such that, for all $R^{\prime} \in P_{R}$, $h(R)=h\left(R^{\prime}\right)$ and for each $R^{\prime}$ there exists $l \in b(R),\left(b\left(R^{\prime}\right) \backslash\{\bar{l}\}\right)=(b(R) \backslash\{l\})$ (resp. $\left.\left(b\left(R^{\prime}\right) \backslash\{\bar{l}\}\right) \subseteq(b(R) \backslash\{l\})\right)$. The full naïve (resp. ground) resolution of $R$ by $P$ is the set of all possible naïve (resp. ground) resolutions of $R$ with the rules of $P: \operatorname{res}_{f}(R, P)=\left\{\operatorname{res}\left(R, R^{\prime}\right) \mid R^{\prime} \in P_{R}\right\}$.

Theorem 1 (Completeness and Soundness of full resolution). Given a set $E$ of pairs of interpretations, LF1T with full naïve (resp. ground) resolution is complete and sound for $E$.

Theorem 2 (LF1T with full resolution learns complete prime NLP). Given a set $E$ of pairs of interpretations, LF1T with full naïve (resp. ground) resolution will learn the complete prime NLP that realizes E.

\subsection{Least Specialization for LF1T}

Until now, to construct an NLP, LF1T relied on a bottom-up method that generates hypotheses by generalization from the most specific clauses or examples until every positive example is covered. This time we propose a new learning method that generate hypotheses by specialization from the most general rules until no negative example is covered. Learning by specialization ensures to output the most general valid hypothesis. that is similar to the notion of specialization we use here amoung body of rules with the same head. In ILP, refinement operators usually apply a substitution $\theta$ and add a set of literals to a clause [13]. Similarly, in our new algorithm, we refine rules by adding the negation of negative transitions into their body.

Definition 9 (Least specialization). We call a maximal specialization of a rule $R$, a rule $R_{S}$ if $h\left(R_{S}\right)=h(R)$ and $b\left(R_{S}\right)$ is a maximal specialization of $b(R)$. Let $R_{1}$ and $R_{2}$ be two rules such that $h\left(R_{1}\right)=h\left(R_{2}\right)$ and $R_{1}$ subsumes $R_{2}$, e.g. $b\left(R_{1}\right) \subseteq b\left(R_{2}\right)$. Let $l_{i}$ be the $i^{\text {th }}$ literal of $b\left(R_{2}\right)$, then the least specialization of $R_{1}$ over $R_{2}$ is as follows:

$l s\left(R_{1}, R_{2}\right)=\left\{h\left(R_{1}\right) \leftarrow\left(b\left(R_{1}\right) \wedge \neg b\left(R_{2}\right)\right)\right\}=\left\{\left(h\left(R_{1}\right) \leftarrow\left(b\left(R_{1}\right) \wedge \overline{l_{i}}\right) \mid l_{i} \in b\left(R_{2}\right) \backslash b\left(R_{1}\right)\right\}\right.$ 
Let $P$ be an NLP, $R$ be a rule and $S$ be the set of all rules of $P$ that subsumes $R$. The least specialization $l s(P, R)$ of $P$ by $R$ is as follow

$$
l s(P, R)=(P \backslash S) \cup\left(\bigcup_{R_{P} \in S} l s\left(R_{P}, R\right)\right)
$$

The least specialization of a rule $R$ can be used to avoid the subsumption of another rule with a minimal reduction of the generality of $R$. The least specialization of an NLP $P$ can be used to avoid the coverage of a negative transition with a minimal reduction of the generality of the rules of $P$.

Theorem 3 (Soundness of least specialization). Let $R_{1}, R_{2}$ be two rules such that $R_{1}$ subsumes $R_{2}$. Let $S_{1}$ be the set of rules subsumed by $R_{1}$ and $S_{2}$ be the rules of $S_{1}$ that subsume $R_{2}$. The least specialization of $R_{1}$ by $R_{2}$ only subsumes the set of rules $S_{1} \backslash S_{2}$. Let $P$ be a NLP and $R$ be a rule such that $P$ subsumes $R$. Let $S_{P}$ be the set of rules subsumed by $P$ and $S_{R}$ be the rules of $S_{P}$ that subsume $R$. The least specialization of $P$ by $R$ only subsumes the set of rules $S_{P} \backslash S_{R}$.

\section{Algorithm}

Now we present a new LF1T algorithm based on least specialization. The novelty of this approach is double: first it relies on specialization in place of generalization and most importantly, it guarantees that the output is the complete prime NLP that realize the input transitions, as shown by Theorem 5. Algorithm 1 shows the pseudo-code of LF1T with least specialization. Like in previous versions,

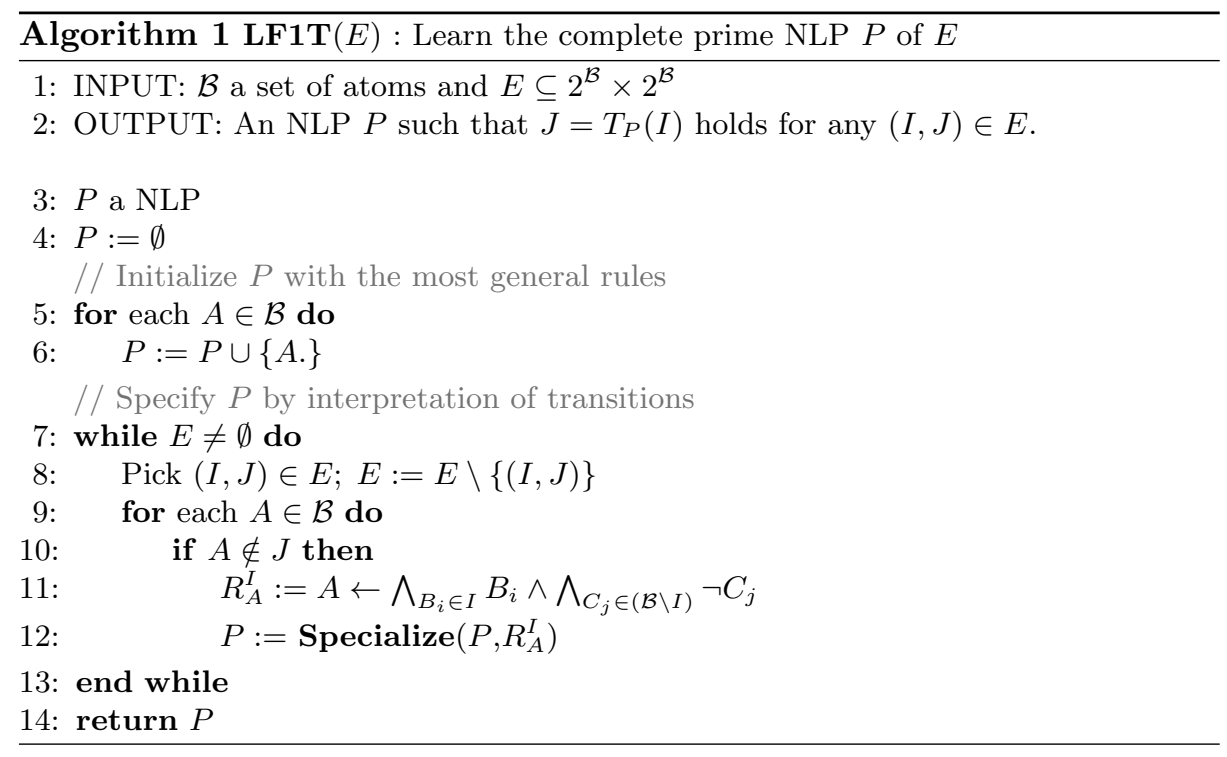


LF1T takes a set of state transitions $E$ as input and outputs an NLP $P$ that realizes $E$. To guarantee the minimality of the learned NLP, LF1T starts with an initial NLP $P_{0}^{\mathcal{B}}$ that is the most general complete prime NLP of the Herbrand base $\mathcal{B}$ of $E$, i.e. the NLP that contains only facts (lines 3-7): $P_{0}^{\mathcal{B}}=\{p \cdot \mid p \in \mathcal{B}\}$. Then LF1T iteratively analyzes each transition $(I, J) \in E$ (lines 8-13).

For each variable $A$ that does not appear in $J$, LF1T infers an anti-rule $R_{A}^{I}$ (lines 11-12):

$$
R_{A}^{I}:=A \leftarrow \bigwedge_{B_{i} \in I} B_{i} \wedge \bigwedge_{C_{j} \in(\mathcal{B} \backslash I)} \neg C_{j}
$$

A is in the head as it denotes a negative example. Then, LF1T uses least specialization to make $P$ consistent with all $R_{A}^{I}$ (line 12). Algorithm 2 shows in detail the pseudo code of this operation. LF1T first extracts all rules $R_{P} \in P$

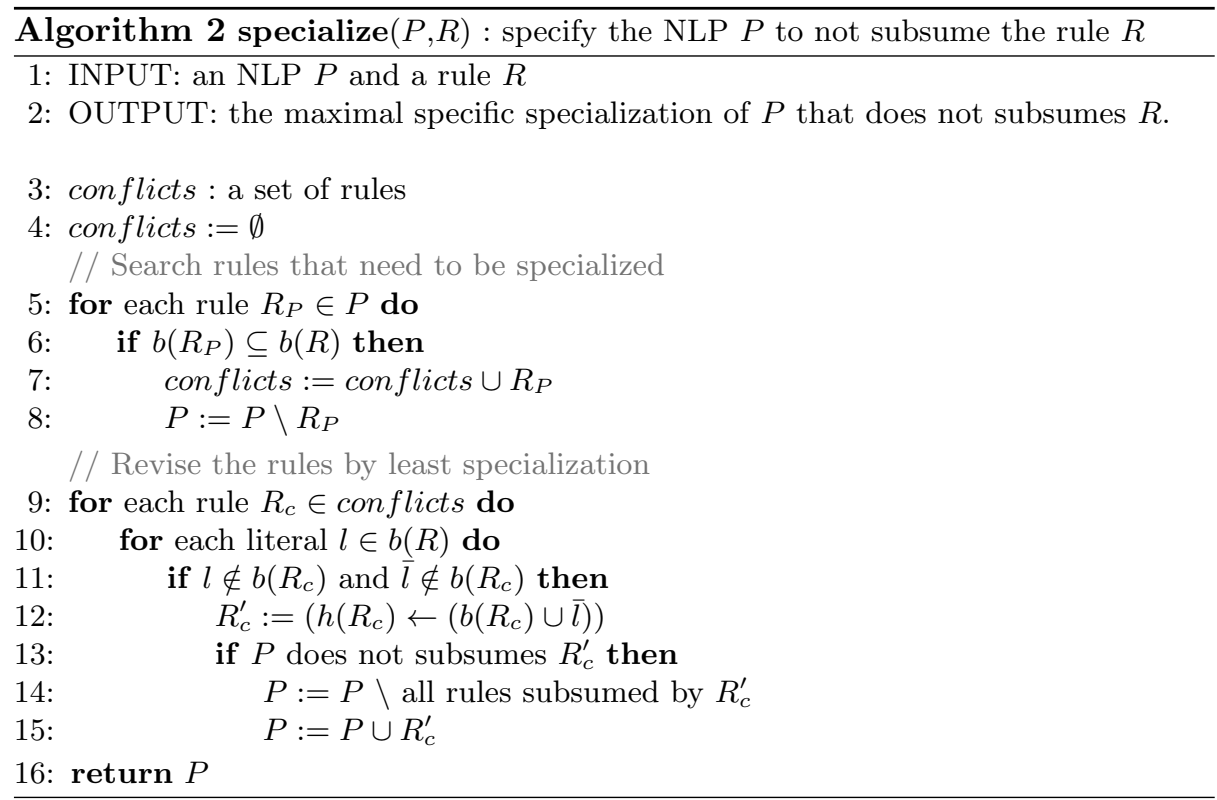

that subsume $R_{A}^{I}$ (lines 3-8). It generates the least specialization of each $R_{P}$ by generating a rule for each literal in $R_{A}^{I}$ (lines 9-12). Each rule contains all literals of $R_{P}$ plus the opposite of a literal in $R_{A}^{I}$ so that $R_{A}^{I}$ is not subsumed by that rule. Then LF1T adds in $P$ all the generated rules that are not subsumed by $P$ (line 13-15), so that $P$ becomes consistent with the transition $(I, J)$ and remains a complete prime NLP. When all transitions have been analyzed, LF1T outputs $P$ that has become the complete prime NLP of $E$.

Table 1 shows the execution of $\mathbf{L F 1 T}$ with least specialization on step transitions of figure 1 where $p q r \rightarrow p q$ represents the state transition $(\{p, q, r\},\{p, q\})$. Introduction of literal by least specialization is represented in bold and rules 
Table 1. Execution of LF1T with least specialization on state transitions of figure 1

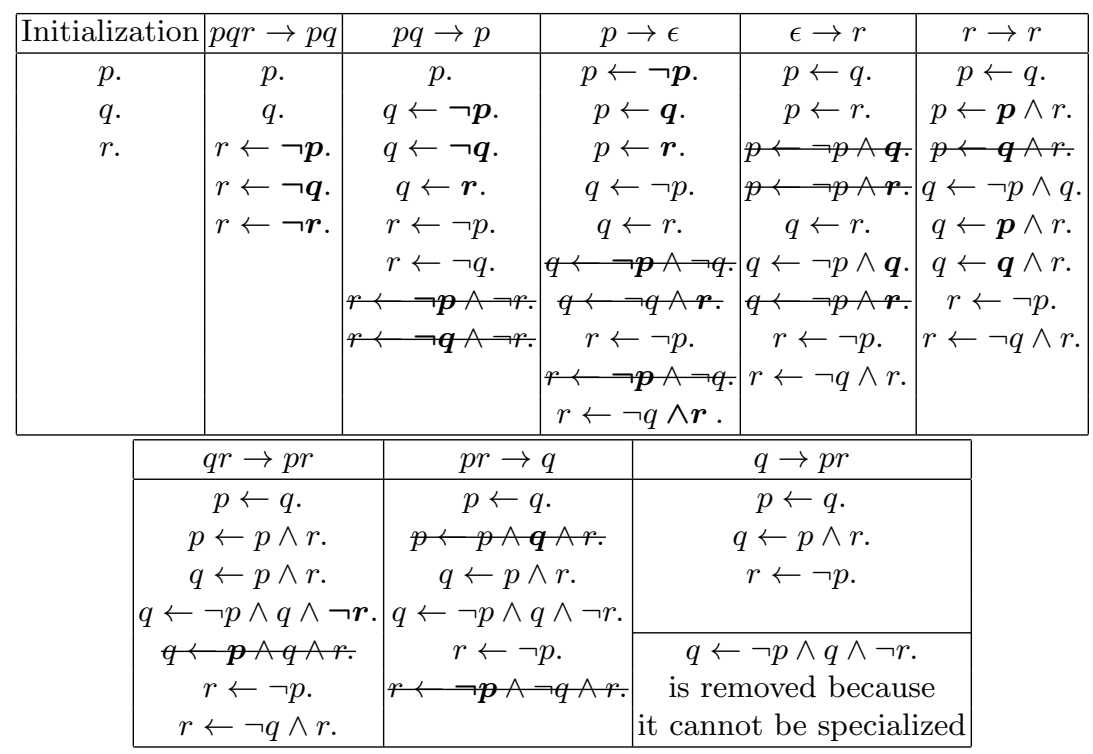

that are subsumed after specialization are stroked. LF1T starts with the most general set of prime rules that can realize $E$, that is $P=\{p ., q ., r$. $\}$. From the transition $(p q r, p q) \mathbf{L F 1 T}$ infers the rule $r \leftarrow p \wedge q \wedge r$ that is subsumed by $r . \in P$. LF1T then replaces that rule by its least specialization: $l s(r ., r \leftarrow p \wedge q \wedge r)=$ $\{r \leftarrow \neg p, r \leftarrow \neg q, r \leftarrow \neg r\}$ Furthermore, $P$ becomes consistent with $(p q r, p q)$. From $(p q, p)$ LF1T infers two rules: $q \leftarrow p \wedge q \wedge \neg r$ and $r \leftarrow p \wedge q \wedge \neg r$, that are respectively subsumed by $q$. and $r \leftarrow \neg r$. The first rule, $q$., is replaced by its least specialization: $\{q \leftarrow \neg p, q \leftarrow \neg q, q \leftarrow r\}$. For the second rule, $r$., its least specialization by $r \leftarrow p \wedge q \wedge \neg r$ generates two rules, $r \leftarrow \neg p \wedge \neg r$ and $r \leftarrow \neg q \wedge \neg r$. But these rules are respectively subsumed by $r \leftarrow \neg p$ and $r \leftarrow \neg q$ that are already in $P$. The subsumed rules are not added to $P$, so that the analysis of $(p q, p)$ results in the specialization of $q$. and the deletion of $r \leftarrow \neg r$.

Learning continues with similar cases until the last transition $(q, p r)$ where we have a special case. From this transition, LF1T infers the rule $q \leftarrow \neg p \wedge q \wedge \neg r$ that is subsumed by $P$ on $R:=q \leftarrow \neg p \wedge q \wedge \neg r$. Because $|b(R)|=|\mathcal{B}|$ it cannot be specialized so that $P$ becomes consistent with $(q, p r)$, LF1T just removes $R$ from $P$.

Theorem 4 (Completeness of LF1T with least specialization). Let $P_{0}^{\mathcal{B}}$ be the most general complete prime NLP of a given Herbrand base B. Initializing $\mathbf{L F 1 T}$ with $P_{0}^{\mathcal{B}}$, by using least specialization iteratively on a set of state transitions $E, \mathbf{L F 1 T}$ learns an $N L P$ that realizes $E$.

Theorem 5 (LF1T with least specialization outputs a complete prime NLP). Let $P_{0}^{\mathcal{B}}$ be the most general complete prime NLP of a given Herbrand base B. Initializing LF1T with $P_{0}^{\mathcal{B}}$, by using least specialization iteratively on a set of state transitions $E, \mathbf{L F 1 T}$ learns the complete prime NLP of E. 
Theorem 6 (Complexity). Let $n$ be the size of the Herbrand base $|\mathcal{B}|$. Using least specialization, the memory complexity of $\mathbf{L F 1 T}$ remains in the same order as the previous algorithms based on ground resolution, i.e., $O\left(2^{n}\right)$. But the computational complexity of LF1T with least specialization is higher than the previous algorithms based on ground resolution, i.e $O\left(n \cdot 4^{n}\right)$ and $O\left(4^{n}\right)$, respectively. Same complexity results for full naïve (resp. ground) resolution.

\section{Evaluation}

In this section, we evaluate our new learning methods through experiments. We apply our new LF1T algorithms to learn Boolean networks. Here we run our learning program on the same benchmarks used in [8] and [16]. These benchmarks are Boolean networks taken from Dubrova and Teslenko [3], which include those networks about control of flower morphogenesis in Arabidopsis thaliana, budding yeast cell cycle regulation, fission yeast cell cycle regulation, mammalian cell cycle regulation and $\mathrm{T}$ helper cell cycle regulation. Like in $[8,16]$, we first construct an NLP $\tau(N)$ from the Boolean function of a Boolean network $N$ where each Boolean function is transformed into a DNF formula. Then, we get all possible 1-step state transitions of $N$ from all $2^{|\mathcal{B}|}$ possible initial states $I^{0}$ 's by computing all stable models of $\tau(N) \cup I^{0}$ using the answer set solver clasp [5]. Finally, we use this set of state transitions to learn an NLP using our LF1T algorithm. Because a run of LF1T returns an NLP which can contain redundant rules, the original NLP $P_{\text {org }}$ and the output NLP $P_{L F I T}$ of LF1T can be different, but remain equivalent with respect to state transition, that is, $T_{P_{\text {org }}}$ and $T_{P_{L F I T}}$ are identical functions. Regarding the new algorithms, it can also be the case if the original NLP is not a complete prime NLP. For the new versions of LF1T, if $P_{\text {org }}$ is not a prime complete NLP we will learn a simplification of $P_{\text {org }}$. Table 2 shows the memory space and time of a single LF1T run in learning a Boolean network for each benchmark on a processor Intel Core I7 (3610QM, $2.3 \mathrm{GHz}$ ) with $4 \mathrm{~GB}$ of RAM. It compares memory and run time of the three previous algorithm (naïve, ground and the BDD optimization of the ground version) with their extension to learn complete prime NLP and the new algorithm based on least specialization. For each version of LF1T the variable ordering is alphabetical and transition ordering is the one that clasp outputs. The time limit is set to two hours for each experiment. Memory is represented in (maximal) number of literal in the NLP learned. Except for LF1T-BDD, all implemented algorithms uses the same data structures. That is why even LF1T with least specialization cannot compete with the ground-BDD version regarding memory and run time. It is more relevant to compare it to the original implementation of LF1T with ground resolution and the new one with full ground resolution.

On Table 2 we can observe that, as the number of variable increases, the memory efficiency of least specialization regarding ground version becomes more interesting. Regarding run time, both algorithms have globally equivalent performances. But least specialization ensure that the output is unique in the fact that it is the complete prime NLP of the given input transitions. LF1T with 


\begin{tabular}{|c|c|c|c|c|c|}
\hline Algorithm & Mammalian (10) & Fission (10) & Budding (12) & Arabidopsis (16) & T helper (23) \\
\hline Naïve & $142118 / 4.62 \mathrm{~s}$ & $126237 / 3.65 \mathrm{~s}$ & $147124 / 523 \mathrm{~s}$ & T.O. & T.O. \\
Ground & $1036 / \mathbf{0 . 0 4}$ & $1218 / \mathbf{0 . 0 5}$ & $21470 / 0.26 \mathrm{~s}$ & $271288 / 4.25 \mathrm{~s}$ & T.O. \\
Ground-BDD & $\mathbf{1 8 0 / 0 . 2 4 s}$ & $\mathbf{1 4 7} / 0.24 \mathrm{~s}$ & $\mathbf{5 4 1 / 0 . 1 9 s}$ & $\mathbf{7 7 9 / 2 . 8 s}$ & $\mathbf{6 1 1} / \mathbf{3 3 6 0 s}$ \\
\hline Full Naïve & $377539 / 29.25 \mathrm{~s}$ & $345587 / 24.03 \mathrm{~s}$ & T.O. & T.O. & T.O. \\
Full Ground & $1066 / 0.24 \mathrm{~s}$ & $1178 / 0.23 \mathrm{~s}$ & $23738 / 4.04 \mathrm{~s}$ & $399469 / 111 \mathrm{~s}$ & T.O. \\
Least Specialization & $\mathbf{3 7 5 / 0 . 0 6 s}$ & $\mathbf{3 7 7} / \mathbf{0 . 0 8} \mathbf{s}$ & $\mathbf{6 4 1} / \mathbf{0 . 3 5 s}$ & $\mathbf{2 2 7 0} / \mathbf{5 . 2 8 s}$ & $\mathbf{3 1 3 4 / 5 2 6 3 s}$ \\
\hline
\end{tabular}

Table 2. Memory use and learning time of LF1T for Boolean networks benchmarks up to 23 nodes in the same condition as in [8]

full ground resolution also ensure this property, but is much less efficient than least specialization regarding both memory use and run time. On the benchmark, least specialization is respectively $75 \%, 65 \%, 91 \%$ and $95 \%$ faster. Least specialization version also succeed to learn the t-helper benchmark (23 variables) in 1 hour and 21 minutes. The main interest of using least specialization is that it guarantees to obtain a unique NLP that contains all minimal conditions to make a variable true. Previous versions of LF1T do not have this property and experimental results showed that their output is sensitive to variable ordering and especially transition ordering. For a given set of state transitions $E$, the output of LF1T with least specialization is always the same whatever the variable ordering or transition ordering. It is easy to see that variable ordering has no impact on both learning time and memory use of the new versions of LF1T since they consider all generalizations/specializations. But transition ordering has a significant impact on the learning time of the new version of LF1T compared to previous ones. On all experiments we run, the ordering of the output of clingo gives the best results. More investigation are required to determine if we can design a heuristic to make a good ordering of the input transition to speed up the run time of the new algorithms.

\section{Conclusion and Future Work}

We proposed a new algorithm for learning from interpretation transitions based on least specialization. Given any state transition diagram we can learn an NLP that exactly captures the system dynamics. Learning is performed only from positive examples, and produces NLPs that consist only of rules to make literals true. Consistency of state transition rules is achieved by least specialization, in which minimality of rules is guaranteed. As a result, given any state transition diagram $E$, LF1T with least specialization always learns a unique NLP that contains all prime rules that realize $E$. It implies that the output of LF1T is no more sensitive to variable ordering or transition ordering. But, experimental results showed that the new algorithm is sensitive to input transitions ordering regarding run time. Design of an heuristic to make a good ordering of the input is one possible future work. We are now considering to extend our framework to learn non-deterministic dynamic systems. One of our expectation is to be able to learn probabilistic logic program from interpretation of transitions. Assuming that probability of transition are given as input it should be possible to infer probabilistic rules using adapted LFIT techniques. But how to combine probabilities when generalization/specialization occurs is an interesting problem that we plan to tackle in our future works. 


\section{References}

1. Apt, K.R., Blair, H.A., Walker, A.: Towards a theory of declarative knowledge. Foundations of deductive databases and logic programming p. 89 (1988)

2. Avila Garcez, A., Zaverucha, G.: The connectionist inductive learning and logic programming system. Applied Intelligence 11(1), 59-77 (1999)

3. Dubrova, E., Teslenko, M.: A sat-based algorithm for finding attractors in synchronous boolean networks. IEEE/ACM Transactions on Computational Biology and Bioinformatics (TCBB) 8(5), 1393-1399 (2011)

4. d'Avila Garcez, A., Broda, K., Gabbay, D.: Symbolic knowledge extraction from trained neural networks: A sound approach. Artificial Intelligence 125(12), 155 - 207 (2001), http://www.sciencedirect.com/science/article/pii/S0004370200000771

5. Gebser, M., Kaminski, R., Kaufmann, B., Schaub, T.: Answer Set Solving in Practice. Synthesis Lectures on Artificial Intelligence and Machine Learning, Morgan and Claypool Publishers (2012)

6. Inoue, K.: Logic programming for boolean networks. In: Proceedings of the Twenty-Second international joint conference on Artificial Intelligence-Volume Volume Two. pp. 924-930. AAAI Press (2011)

7. Inoue, K.: Dnf hypotheses in explanatory induction. In: Inductive Logic Programming, pp. 173-188. Springer (2012)

8. Inoue, K., Ribeiro, T., Sakama, C.: Learning from interpretation transition. Machine Learning pp. 1-29 (2012)

9. Inoue, K., Sakama, C.: Oscillating behavior of logic programs. In: Correct Reasoning, pp. 345362. Springer (2012)

10. Kean, A., Tsiknis, G.: An incremental method for generating prime implicants/implicates. Journal of Symbolic Computation 9(2), 185-206 (1990)

11. Michalski, R.S.: A theory and methodology of inductive learning. Artificial intelligence 20(2), 111-161 (1983)

12. Mitchell, T.M.: Generalization as search. Artificial intelligence 18(2), 203-226 (1982)

13. Muggleton, S., De Raedt, L.: Inductive logic programming: Theory and methods. The Journal of Logic Programming 19, 629-679 (1994)

14. Muggleton, S., De Raedt, L., Poole, D., Bratko, I., Flach, P., Inoue, K., Srinivasan, A.: Ilp turns 20. Machine learning 86(1), 3-23 (2012)

15. Plotkin, G.D.: A note on inductive generalization. Machine intelligence 5(1), 153-163 (1970)

16. Ribeiro, T., Inoue, K., Sakama, C.: A bdd-based algorithm for learning from interpretation transition. to appear in LNAI (2013), presented at the 23rd International Conference on Inductive Logic Programming (ILP 2013)

17. Tison, P.: Generalization of consensus theory and application to the minimization of boolean functions. Electronic Computers, IEEE Transactions on (4), 446-456 (1967)

18. Van Emden, M.H., Kowalski, R.A.: The semantics of predicate logic as a programming language. Journal of the ACM (JACM) 23(4), 733-742 (1976)

\section{A Appendix}

\section{A.1 Proof of Theorem 1 (completeness)}

Given a set $E$ of pairs of interpretations, LF1T with full naïve (resp. ground) resolution is complete for $E$.

Proof. According to Theorem 1 (resp. 2) of [8], LF1T with naïve (resp. ground) resolution is complete for $E$. It is trivial that any rules produced by naïve (resp. ground) resolution can be obtained by full naïve (resp. ground) resolution. Then, if $P$ and $P^{\prime}$ are respectively obtained by naïve (resp. ground) resolution and full naïve (resp. ground) resolution, $P^{\prime}$ theory-subsumes $P$. If a program $P$ is complete for $E$, a program $P^{\prime}$ that theory-subsumes $P$ is also complete for $E$. Since $P$ is complete for $E$ by Theorem 1 of [8], $P^{\prime}$ is complete for $E$. $\square$

\section{A.2 Proof of Theorem 1: (soundness)}

Given a set $E$ of pairs of interpretations, LF1T with full naïve (resp. ground) resolution is sound for $E$.

Proof. All rules that can be produced by naïve (resp. ground) resolution can be obtained by full naïve (resp. ground) resolution. Since all rules produced by naïve (resp. ground) resolution are sound for $E$ (Corrollary 1 (resp. 2) of [8]), full naïve (resp. ground) resolution is sound for E. $\square$ 


\section{A.3 Proof of Theorem 2}

Given a set $E$ of pairs of interpretations, LF1T with full naïve (resp. ground) resolution learn the complete prime NLP that realize $E$.

Proof. Let us assume that LF1T with full naïve resolution does not learn a prime NLP of $E$. If our assumption is correct it implies that there exists $R$ a prime rule for $E$ that cannot be learned by LF1T with full naïve resolution. Let $\mathcal{B}$ be the herbrand base of $E$.

Case 1: $|b(R)|=|\mathcal{B}|, R$ will be directly infer from a transition $(I, J) \in E$. This is a contradiction with our assumption.

Case 2: $|b(R)|<|\mathcal{B}|$. let $l$ be a literal such that $l \notin b(R)$, according to our assumption, their is a rule $R^{\prime}$ that is one of the rule $R_{1}:=h(R) \leftarrow b(R) \cup l$ or $R_{2}:=h(R) \leftarrow b(R) \cup \bar{l}$ and $R^{\prime}$ cannot be learned because $\operatorname{res}\left(R_{1}, R_{2}\right)=R$. Recursively, what applies to $R$ applies to $R^{\prime}$ until we reach a rule $R^{\prime \prime}$ such that $\left|b\left(R^{\prime \prime}\right)\right|=|\mathcal{B}|$. Our assumption implies that this rule $R^{\prime \prime}$ cannot be learned, but $R^{\prime \prime}$ will be directly infer from a transition $(I, J) \in E$, this is a contradiction. Since ground resolution can learn all rules learn by naïve resolution, the proof also applies to LF1T with full ground resolution. $\square$

\section{A.4 Proof of Theorem 3}

Let $R_{1}, R_{2}$ be two rules such that $b\left(R_{1}\right) \subseteq b\left(R_{2}\right)$. Let $S_{1}$ be the set of rules subsumed by $R_{1}$ and $S_{2}$ be the rules of $S_{1}$ that subsume $R_{2}$. The least specialization of $R_{1}$ by $R_{2}$ only subsumes the set of rules $S_{1} \backslash S_{2}$.

Proof. :

According to Definition 9 , the least specialization of $R_{1}$ by $R_{2}$ is as follows:

$$
l s\left(R_{1}, R_{2}\right)=\left\{h\left(R_{1}\right) \leftarrow\left(b\left(R_{1}\right) \wedge \neg b\left(R_{2}\right)\right)\right\}
$$

All rule $R$ of $S_{2}$ subsumes $R_{2}$, then according to Definition $1 b(R) \subseteq b\left(R_{2}\right)$. If $l s\left(R_{1}, R_{2}\right)$ subsumes an $R$ then there exists $R^{\prime} \in l s\left(R_{1}, R_{2}\right)$ and $b\left(R^{\prime}\right) \subseteq b(R)$. Since $R^{\prime} \in l s\left(R_{1}, R_{2}\right)$, there is a $l \in b\left(R_{2}\right)$ such that $\bar{l} \in b\left(R^{\prime}\right)$, so that $b\left(R^{\prime}\right) \not \subset b\left(R_{2}\right)$. Since all $R \in S_{2}$ subsume $R_{2}$, $R^{\prime}$ cannot subsume any $R$ since $R^{\prime}$ does not subsume $R_{2}$.

Conclusion 1: the least specialization of $R_{1}$ by $R_{2}$ cannot subsume any $R \in S_{2}$.

Let us suppose there is a rule $R^{\prime} \in S_{1}$ that does not subsumes $R_{2}$ and is not subsumed by $l s\left(R_{1}, R_{2}\right)$. Let $l_{i}$ be the $i^{\text {th }}$ literal of $b\left(R_{2}\right)$, then:

$$
l s\left(R_{1}, R_{2}\right)=\left\{\left(h\left(R_{1}\right) \leftarrow\left(b\left(R_{1}\right) \wedge \overline{l_{i}}\right) \mid l_{i} \in b\left(R_{2}\right) \backslash b\left(R_{1}\right)\right\}(1)\right.
$$

$R^{\prime}$ is subsumed by $R_{1}$, so that $R^{\prime}=h\left(R_{1}\right) \leftarrow b\left(R_{1}\right) \cup S$, with $S$ a set of literal. $R^{\prime}$ does not subsume $R_{2}$, so that there exists a $l \in b\left(R_{2}\right) \backslash b\left(R_{1}\right)$ such that $\bar{l} \in S$. According to (1), the rule $R^{\prime \prime}=h\left(R_{1}\right) \leftarrow b\left(R_{1}\right) \wedge \bar{l}$ is in $l s\left(R_{1}, R_{2}\right)$. Since $R^{\prime \prime}$ subsumes $R^{\prime}$ and $R^{\prime \prime} \in l s\left(R_{1}, R_{2}\right)$, $l s\left(R_{1}, R_{2}\right)$ subsumes $R^{\prime}$.

Conclusion 2: the least specialization of $R_{1}$ by $R_{2}$ subsumes all rule of $S_{1}$ that does not subsume $R_{2}$

Final conclusion: the least specialization of $R_{1}$ by $R_{2}$ only subsumes $S_{1} \backslash S_{2}$. $\square$

Now, let $P$ be an NLP and $R$ be a rule such that $P$ subsumes $R$. Let $S_{P}$ be the set of rules subsumed by $P$ and $S_{R}$ be the rules of $S_{P}$ that subsume $R$. The least specialization of $P$ by $R$ only subsumes the set of rules $S_{P} \backslash S_{R}$.

Proof. :

According to Definition 5, the least specialization $l s(P, R)$ of $P$ by $R$ is as follows:

$$
l s(P, R)=\left(P \backslash S_{P}\right) \cup\left(\bigcup_{R_{P} \in S_{P}} l s\left(R_{P}, R\right)\right)
$$

For any rule $R_{P}$ let $S_{R_{P}}$ be the set of rules subsumed by $R_{P}$ and $S_{R_{P} 2} \in S_{R}$ be the rule of $S_{R_{P}}$ that subsume $R$.

According to Theorem 3 the least specialization of $R_{P}$ by $R$ only subsumes $S_{R_{P}} \backslash S_{R_{P} 2}$. So that $\bigcup_{R_{P} \in S_{P}} l s\left(R_{P}, R\right)$ only subsumes $\left(\bigcup_{R_{P} \in S_{P}} S_{R_{P}} \backslash S_{R_{P} 2}\right)=\left(\bigcup_{R_{P} \in S_{P}} S_{R_{P}}\right) \backslash S_{R}$. Then $l s(P, R)$ 
only subsumes the rules subsumed by $\left(P \backslash S_{P}\right) \cup\left(\bigcup_{R_{P} \in S_{P}} S_{R_{P}}\right) \backslash S_{R}$, that is $S_{P} \backslash S_{R}$.

Conclusion: The least specialization of $P$ by $R$ only subsumes $S_{P} \backslash S_{R}$. $\square$

\section{A.5 Proof of Theorem 4}

Let $P_{0}^{\mathcal{B}}$ be the most general complete prime NLP of a given Herbrand base B, i.e. the NLP that contains only facts

$$
P_{0}^{\mathcal{B}}=\{p . \mid p \in \mathcal{B}\}
$$

Initializing LF1T with $P_{0}^{\mathcal{B}}$, by using least specialization iteratively on the transitions of a set of state transitions $E, \mathbf{L F} 1 \mathbf{T}$ learns an NLP $P$ that realizes $E$.

Proof. :

Let $P$ be an NLP consistent with a set of transitions $E^{\prime}, S_{P}$ be the set of rules subsumed by $P$ and a state transition $(I, J)$ such that $E^{\prime} \subset E$ and $(I, J) \in E$ but $(I, J) \notin E^{\prime}$. According to Theorem 3, for any rule $R_{A}^{I}$ that can be inferred by $\mathbf{L F 1 T}$ from $(I, J)$ that is subsumed by $P$, the least specialization $l s\left(P, R_{A}^{I}\right)$ of $P$ by $R_{A}^{I}$ exactly subsumes the rules subsumed by $P$ except the ones subsumed by $R_{A}^{I}$. Since $\left|R_{A}^{I}\right|$ is $|\mathcal{B}|, R_{A}^{I}$ only subsumes itself so that $l s(P, R)$ exactly subsumes $S_{P} \backslash R_{A}^{I}$. Let $P^{\prime}$ be the NLP obtained by least specialization of $P$ with all $R_{A}^{I}$ that can be inferred from $(I, J)$, then $P^{\prime}$ is consistent with $E^{\prime} \cup\{(I, J)\}$.

Conclusion 1: LF1T keep the consistency of the NLP learned.

LF1T start with $P_{0}^{\mathcal{B}}$ as initial NLP. $P_{0}^{\mathcal{B}}$ is at least consistent with $\emptyset \subseteq E$. According to conclusion 1, initializing LF1T with $P_{0}^{\mathcal{B}}$ and by using least specialization iteratively on the element of $E$ when its needed, LF1T learns an NLP that realizes $E$. $\square$

\section{A.6 Proof of Theorem 5}

Let $P_{0}^{\mathcal{B}}$ be the most general complete prime NLP of a given Herbrand base B, i.e. the NLP that contains only facts

$$
P_{0}^{\mathcal{B}}=\{p . \mid p \in \mathcal{B}\}
$$

Initializing LF1T with $P_{0}^{\mathcal{B}}$, by using least specialization iteratively on a set of state transitions $E$, LF1T learns the complete prime NLP of $E$.

Proof. :

Let us assume that LF1T with least specialization does not learn a prime NLP of $E$. If our assumption is correct, according to Theorem $4, \mathbf{L F 1 T}$ learns a NLP $P$, that is consistent with $E$ and $P$ is not the complete prime NLP of $E$. LF1T start with $P_{0}^{\mathcal{B}}$ as initial NLP, $P_{0}^{\mathcal{B}}$ is the most general complete prime NLP that can cover $E$.

Consequence 1: LF1T with least specialization can transform a complete prime NLP into an NLP that is not a complete prime NLP.

Let $P$ be the complete prime NLP of a set of state transition $E^{\prime} \subset E$ and $(I, J) \notin E^{\prime}$, such that $P$ is not consistent with $(I, J)$. Our assumption implies that the least specialization $P^{\prime}$ of $P$ by the rules inferred from $(I, J)$ is not the complete prime NLP of $E^{\prime} \cup(I, J)$. According to Definition 7, there is two possibilities:

- case 1: $\exists R \in P^{\prime}$ such that $R$ is not a prime rule of $E^{\prime} \cup(I, J)$.

- case 2: $\exists R^{\prime} \notin P^{\prime}$ such that $R^{\prime}$ is a prime rule of $E^{\prime} \cup(I, J)$.

Case 1.1: If $R \in P$, it implies that $R$ is a prime rule of $E^{\prime}$ and that $R$ is consistent with $(I, J)$, otherwise $R$ should have been specialized. Because $R$ is not a prime rule of $E^{\prime} \cup(I, J)$ it implies that there exists a rule $R_{m}$ consistent with $E^{\prime} \cup(I, J)$ that is more general than $R$, i.e. $b\left(R_{m}\right) \subset b(R)$. Then $R_{m}$ is also consistent with $E^{\prime}$, but since $R$ is a prime rule of $E^{\prime}$ there does not exist any rule consistent with $E^{\prime}$ that is more general than $R$. This is a contradiction.

Case 1.2: Now let us suppose that $R \notin P$; then $R$ has been obtained by least specialization of a rule $R_{P} \in P$ by a rule inferred from $(I, J)$. It implies that $\exists l \in b(R)$ and $\bar{l} \in I$. If $R$ is not a prime rule of $E^{\prime} \cup(I, J)$, there exists $R_{m}$ a prime rule of $E^{\prime} \cup(I, J)$ and $R_{m}$ is more general than $R$. It implies that $l \in R_{m}$ otherwise $R_{m}$ is not consistents with $(I, J)$ because it will also subsumes $R_{P}$ that is not consistents with $(I, J)$. Since $R_{m}$ is consistent with $E^{\prime} \cup(I, J)$ it is also consistent with $E^{\prime}$. This implies that $\exists R_{m}^{\prime}$ a prime rule of $E^{\prime}$ that subsumes $R_{m}$ (it can be $R_{m}$ itself), $R_{m}^{\prime}$ also subsumes $R$. Since $P$ is the complete prime NLP of $E^{\prime}, R_{m}^{\prime} \in P$. 
Case 1.2.1: Let suppose that $l \notin b\left(R_{m}^{\prime}\right)$, since $l \in b(R)$ and $R_{m}^{\prime}$ subsumes $R$ then $R_{m}^{\prime}$ subsumes $R_{P}$ because $R=h\left(R_{P}\right) \leftarrow b\left(R_{P}\right) \cup l$. But since $R_{P}$ is a prime rule of $E^{\prime}$ it implies that $R_{m}^{\prime}=R_{P}$. In that case it means that $R_{P}$ subsumes $R_{m}$ and since $l \in R_{m}, h\left(R_{P}\right) \leftarrow b\left(R_{P}\right) \cup l$ also subsumes $R_{m}$. Since $h\left(R_{P}\right) \leftarrow b\left(R_{P}\right) \cup l$ is $R, R$ subsumes $R_{m}$ and $R_{m}$ can neither be more general than $R$ nor a prime rule of $E^{\prime} \cup(I, J)$. This is a contradiction with case 2 .

Case 1.2.2: Finally let us suppose that $l \in b\left(R_{m}^{\prime}\right)$, since $R_{m}^{\prime}$ is consistent with $E$ and $l \in I, R_{m}^{\prime}$ is consistent with $E^{\prime} \cup(I, J)$. But $R_{m}^{\prime}$ subsumes $R_{m}$ and since $R_{m}$ is a prime rule of $E^{\prime} \cup(I, J)$ it implies that $R_{m}^{\prime}=R_{m}$. In that case $R_{m} \in P$ and because $R_{m}$ is consistent with $(I, J)$ and $R_{m}$ subsumes $R, \mathbf{L F 1 T}$ will not add $R$ into $P^{\prime}$. This is a contradiction with case 1 .

Case 2: Let consider that there exists a $R^{\prime} \notin P^{\prime}$ such that $R^{\prime}$ is a prime rule of $E^{\prime} \cup(I, J)$. Since $R^{\prime} \notin P^{\prime}, R^{\prime} \notin P$ and $R^{\prime}$ is not a prime rule of $E^{\prime}$ since $P$ is the complete prime NLP of $E^{\prime}$. Then, there exists $R_{m} \in P$ a prime rule of $E^{\prime}$ such that $R_{m}$ subsumes $R^{\prime}$ and $R_{m} \notin P^{\prime}$ since $R^{\prime}$ is a prime rule of $E^{\prime} \cup(I, J)$. Then, $b\left(R^{\prime}\right)=b\left(R_{m}^{\prime}\right) \cup S$ with $S$ a non-empty set of literals such that for all $l \in S, l \notin b\left(R_{m}\right)$. Since $R_{m} \notin P^{\prime}$, there is a rule $R_{h\left(R_{m}\right)}^{I}$ that can be inferred from $(I, J)$ and subsumed by $R_{m}$. And there is no rule $R_{m}^{\prime} \in l s\left(R_{m}, R_{h\left(R_{m}\right)}^{I}\right)$ that subsumes $R^{\prime}$ since $R^{\prime}$ is a prime rule of $E^{\prime} \cup(I, J)$. Then, for all $l^{\prime} \in b\left(R_{h\left(R_{m}\right)}^{I}\right), \overline{l^{\prime}} \notin b\left(R^{\prime}\right)$ otherwise there is a $R_{m}^{\prime}$ that subsumes $R^{\prime}$. Since $\left|b\left(R_{h\left(R_{m}\right)}^{I}\right)\right|=\mathcal{B}, b\left(R^{\prime}\right)$ cannot contains a literal that is not in $b\left(R_{h\left(R_{m}\right)}^{I}\right)$ so that $R^{\prime}$ subsumes $R_{h\left(R_{m}\right)}^{I}$. $R^{\prime}$ cannot be a prime rule of $E^{\prime} \cup(I, J)$ since $R^{\prime}$ is not consistent with $(I, J)$, this is a contradiction.

Conclusion: If $P$ is a complete prime NLP of $E^{\prime} \subset E$, for any $(I, J) \in E \mathbf{L F 1 T}$ with least specialization will learn the complete prime NLP $P^{\prime}$ of $E^{\prime} \cup(I, J)$. Since LF1T starts with a complete prime NLP that is $P_{0}^{\mathcal{B}}$, according to Theorem 4 , LF1T will learn a NLP consistent with $E$, our last statement implies that this NLP is the complete prime NLP of $E$ since LF1T cannot specify a complete prime NLP into an NLP that is not a complete prime NLP. $\square$

\section{A.7 Proof of Theorem 6}

Let $n$ be the size of the Herbrand base $|\mathcal{B}|$. Using least specialization, the memory complexity of LF1T remains in the same order as the previous algorithms based on ground resolution, i.e., $O\left(2^{n}\right)$. But the computational complexity of LF1T with least specialization is higher than the previous algorithms based on ground resolution, i.e $O\left(n \cdot 4^{n}\right)$ and $O\left(4^{n}\right)$, respectively. Same complexity results for full naïve (resp. ground) resolution.

Proof. Let $n$ be the size of the Herbrand base $|\mathcal{B}|$ of a set of state transitions $E$. This $n$ is also the number of possible heads of rules. Furthermore, $n$ is also the maximum size of a rule, i.e. the number of literals in the body; a literal can appear at most one time in the body of a rule. For each head there are $3^{n}$ possible bodies: each literal can either be positive, negative or absent from the body. From these preliminaries we conclude that the size of a NLP $|P|$ learned by LF1T from $E$ is at most $n \cdot 3^{n}$. But since a NLP $P$ learned by LF1T only contains prime rules of $E,|P|$ cannot exceed $n \cdot 2^{n}$; in the worst case, $P$ contains only rules of size $n$ where all literals appear and there is only $n \cdot 2^{n}$ such rules. If $P$ contains a rule with $m$ literals $(m<n)$, this rule subsumes $2^{n-m}$ rules which cannot appear in $P$. Finally, least specialization also ensures that $P$ does not contain any pair of complementary rules, so that the complexity is further divided by $n$; that is, $|P|$ is bounded by $O\left(\frac{n \cdot 2^{n}}{n}\right)=O\left(2^{n}\right)$.

When LF1T infers a rule $R_{A}^{I}$ from a transition $(I, J) \in E$ where $A \notin J$, it has to compare it with all rules in $P$ to extract the ones that need to be specialized. This operation has a complexity of $O(|P|)=O\left(2^{n}\right)$. Since $\left|b\left(R_{A}^{I}\right)\right|=n$, according to Definition 5 the least specialization of a rule $R \in P$ can at most generate $n$ different rules. In the worst case all rules of $P$ with $\overline{h\left(R_{A}^{I}\right)}$ as head subsume $R_{A}^{I}$. There are possibly $2^{n} / n$ such rules in $P$, so that LF1T generates at most $2^{n}$ rules for each $R_{A}^{I}$. For each $(I, J) \in E$, LF1T can infer at most $n$ rules $R_{A}^{I}$. In the worst case, LF1T can generates $n \cdot 2^{n}$ rules that are compared with the $2^{n}$ rules of $P$. Thus, construction of an NLP which realizes $E$ implies $n \cdot 2^{n} \cdot 2^{n}=n \cdot 4^{n}$ operations. The same proof applies to LF1T naïve (resp. ground) resolution, when LF1T infers a rule $R_{A}^{I}$ from a transition $(I, J) \in E$ where $A \in J$. The complexity of learning an NLP from a complete set of state transitions with an Herbrand base of size $n$ is $O\left(n \cdot 4^{n}\right)$. $\square$ 